\title{
ASSESSMENT OF THE UTILITY OF THE ADVANCED HIMAWARI IMAGER TO DETECT ACTIVE FIRE OVER AUSTRALIA
}

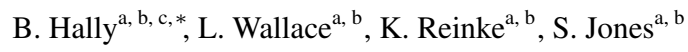 \\ ${ }^{\text {a }}$ School of Mathematics and Geospatial Science, RMIT University, Melbourne, Australia \\ ${ }^{\mathrm{b}}$ Bushfire and Natural Hazards Cooperative Research Centre, East Melbourne, Australia \\ ${ }^{\mathrm{c}}$ Faculty for Geo-Information Science and Earth Observation (ITC), University of Twente, Enschede, Netherlands
}

Commission VIII, WG VIII/1

KEY WORDS: Himawari, fire detection, thermal imaging, temporal analysis, Kalman filtering

\begin{abstract}
:
Wildfire detection and attribution is an issue of importance due to the socio-economic impact of fires in Australia. Early detection of fires allows emergency response agencies to make informed decisions in order to minimise loss of life and protect strategic resources in threatened areas. Until recently, the ability of land management authorities to accurately assess fire through satellite observations of Australia was limited to those made by polar orbiting satellites. The launch of the Japan Meteorological Agency (JMA) Himawari-8 satellite, with the 16-band Advanced Himawari Imager (AHI-8) onboard, in October 2014 presents a significant opportunity to improve the timeliness of satellite fire detection across Australia. The near real-time availability of images, at a ten minute frequency, may also provide contextual information (background temperature) leading to improvements in the assessment of fire characteristics. This paper investigates the application of the high frequency observation data supplied by this sensor for fire detection and attribution. As AHI-8 is a new sensor we have performed an analysis of the noise characteristics of the two spectral bands used for fire attribution across various land use types which occur in Australia. Using this information we have adapted existing algorithms, based upon least squares error minimisation and Kalman filtering, which utilise high frequency observations of surface temperature to detect and attribute fire. The fire detection and attribution information provided by these algorithms is then compared to existing satellite based fire products as well as in-situ information provided by land management agencies. These comparisons were made Australia-wide for an entire fire season including many significant fire events (wildfires and prescribed burns). Preliminary detection results suggest that these methods for fire detection perform comparably to existing fire products and fire incident reporting from relevant fire authorities but with the advantage of being near-real time. Issues remain for detection due to cloud and smoke obscuration, along with validation of the attribution of fire characteristics using these algorithms.
\end{abstract}

\section{INTRODUCTION}

The use of remote sensing satellites for detecting and imaging natural disasters has steadily increased with the availability and reliability of sensors in orbit. The ability of emergency responders to view objective information about the location, size and impact of an incident enables timely response and accurate assessment, with benefits such as advanced warning of the impending event, the ability to prioritise resources to mitigate impact where feasible, and enabling planning of relief measures in the aftermath of an event. Remotely sensed information can also be tied to models to simulate the incident at hand, which allows for more accurate estimates of the change of an incident over time.

A common way to determine sudden change in an environment caused by an external influence can be through the use of thermal infra red imaging. Changes to the thermal signature of the land surface can be caused by a number of disaster types - flooding will cause a flattening of temperature response in a diurnal cycle, with cooler high temperatures and warmer minimums, and fire will cause temperatures to increase, with a significant impact in the medium wave infra-red due to fire temperatures and their corresponding blackbody radiation outputs. These changes can occur rapidly with the spread of the event, so they can easily be used to determine the extents of the affected area.

\footnotetext{
${ }^{*}$ Corresponding author - bryan.hally@ rmit.edu.au
}

\subsection{Identifying fire}

Traditional methods for the detection and monitoring of fire events have generally consisted of in-situ fire detection personnel and reports from members of the public. These methods tend to detect the initial fire activity very quickly as smoke from fire events can be spotted easily from a distance. Shortcomings of these methods include where a fire is in a remote area or in rugged terrain, and where weather conditions such as cloud mask visual identification of smoke. These information sources also provide poor information of the ongoing nature of fire events - subjective reports of fire extent and intensity can vary between observers, and terrain and vegetation may mask observation of the full extent of fire through over or under reporting. Monitoring of fire events can also take place from airborne platforms, and while these are very effective at collecting information about fire extent, the availability of aerial devices, and the cost and risk involved to personnel and equipment can limit or preclude their use in many situations.

The use of satellite sensors to provide remotely sensed images of fire can address some of these shortcomings. By their nature, satellite sensors provide a more global coverage of fire activity, with little difference in detection capability due to remoteness or inaccessibility (Roy et al., 2013). The sensor provides objective information about fire activity at a given time - the fire size and intensity information derived from imagery is superior to subjective assessment of fire. Using remote sensing also reduces the infrastructure, assets and personnel required to monitor fire activity, with an associated reduction of risk and cost. With the use of a range of different sensors, ongoing monitoring of fire activ- 
ity and spread can occur, leading to better decisions about the mitigation of fire along with disaster relief planning.

Current algorithms for the detection of fire in the environment are based on either empirical thresholds applied to brightness temperatures of middle infra red bands of satellite sensors, or more complicated contextual algorithms based on the comparison of potential fire pixels to their surrounds (Roy et al., 2013). Currently the use of contextual algorithms is considered state of the art, and when tuned correctly produce significantly higher rates of detection, especially for smaller, low intensity incidents $(\mathrm{Li}$ et al., 2013). Contextual algorithms for fire rely on deriving the fire pixel's radiant output in comparison to what the equivalent background temperature output should be, generally based upon the brightness temperature of surrounding pixels. A number of factors can lead these techniques to producing less than desirable results - surrounding pixels can be fire affected in themselves, spreading the area required to acquire fire-free pixels for the algorithm calculation; the point spread of the sensor can lead to false detection in adjacent pixels; and the adjacent pixels used to calculate the theoretical background temperature of the target pixel may have significant differences in landform, surface emissivity, land cover percentage and solar reflectivity, leading to large differences between the actual and calculated fire background temperatures from these algorithms.

\subsection{Himawari AHI-8 sensor}

The Japan Meteorological Agency (JMA) launched the Himawari8 geostationary satellite, the latest in their line of MTSAT platforms, in October 2014. The sensor onboard this platform is the Advanced Himawari Imager (AHI-8), which delivers 16 bands of imaging information at ten minute intervals, and is used primarily for weather forecasting and monitoring. The sensor has coverage of the area bounded by western India, Hawaii, Eastern Antarctica and Eastern Siberia. Generally there are two housekeeping timeslots for each day, at 0240 and 1440 UTC, meaning a maximum of 142 images are obtained on any one day. Station keeping for navigation and image calibration purposes occurs on a regular basis, with announcements of these activities on the Japan Meteorological Agency mission website.

Of importance for event monitoring are the many bands of this sensor located in the medium wave and thermal infrared, along with visible and near-infrared imagery, which is supplied at a spatial resolution of $2 \mathrm{~km}$ at nadir $(0.5 \mathrm{~km}$ for one visible channel). This is a vast improvement of performance compared to the previous sensor MTSAT-2, both in the spatial and spectral resolution, and refresh rate. The sensor also has far higher saturation temperatures in the medium wave infrared Band 7, which enables the attribution of larger fires than its predecessor, along with greater radiometric depth and higher signal to noise ratios for the corresponding bands. Images have been available from this sensor since JMA switched from MTSAT-2 in July 2015. A comparison showing the improved abilities of AHI-8 compared to MTSAT-2 can be found in Table 1 .

This ability to monitor change rapidly increases with the use of geostationary imagers, which can provide near real-time updates of incidents from a fixed viewpoint. Most remotely sensed images used for this kind of disaster mapping and planning in the past have come from low earth orbiting satellites. These have the advantage of being much closer to the earth's surface, with the benefits of a higher spatial and radiometric resolution than geostationary satellites viewing the same scenes. The major disadvantage of the low earth orbit systems in use are their temporal availability - the systems currently in place can supply imagery
Table 1: Comparison of MTSAT-2 and AHI-8 sensors for fire detection

\begin{tabular}{|l|l|l|}
\hline Sensor & MTSAT-2 & AHI-8 \\
\hline Number of bands & 5 & 16 \\
Temporal Resolution & $30 \mathrm{~min}$ & $10 \mathrm{~min}$ \\
Spatial Resolution (nadir) & $4 \times 4 \mathrm{~km}$ & $2 \times 2 \mathrm{~km}$ \\
$\begin{array}{l}\text { Medium wave infra-red channel } \\
\text { saturation temperature }\end{array}$ & $330 \mathrm{~K}$ & $400 \mathrm{~K}$ \\
$\begin{array}{l}\text { Quantisation (MWIR) } \\
\text { Noise equivalent delta tempera- } \\
\text { ture }(N E \Delta T)\end{array}$ & $10 \mathrm{bit}$ & \\
& $0.09 @ \mathrm{bit}$ \\
\hline
\end{tabular}

at a revisit rate of no better than four hours, leaving large gaps in the supply of images, especially for highly dynamic systems such as fire. The use of geostationary sensors with a higher revisit rate can overcome the issues caused by lower spatial and radiometric resolution by simply supplying data more often. Geostationary sensors also have the advantage of viewing incidents from the same viewpoint over time, meaning less geometric correction of images is required, leading to far easier analysis of change over time.

All of these improvements in sensor ability and availability contribute to improved ability to image change in the environment and monitor it in near real-time. The AHI- 8 sensor is also similar in construction to the ABI system that will be deployed on the third generation GOES satellite platform, which is due for its first launch in October 2016, and initial findings from the images produced by AHI- 8 will be able to be applied almost directly to ABI imagery from launch.

This paper looks to evaluate the ability of the AHI-8 sensor to detect initial fire activity in the environment, with a specific focus on the application of a temporal-based algorithm, rather than the current contextual-based algorithms used by polar orbiting sensors. The paper will describe the importance of background temperature in the determination of fire characteristics, introduce a new method of defining background temperature for a specific pixel, and demonstrate some initial findings from its application.

\section{LAND SURFACE TEMPERATURE DETERMINATION}

Derivation of fire attributes from remotely sensed images can occur using either of two methods. The method of bi-spectral retrieval of fire characteristics was first proposed by (Dozier, 1981), which uses differences in the radiometric returns of a medium wave infra red and thermal infra red channel to determine the fire size and intensity. The other method used for fire characterisation is the calculation of fire radiative power, proposed by (Kaufman et al., 1998) and refined by (Wooster et al., 2003), which uses a number of thermal components of a pixel to generate total fire energy output. Both of these methods rely on an accurate measure of the background temperature of the analysed pixel. A study by (Giglio and Kendall, 2001) highlighted issues with fire retrievals using the bi-spectral method, especially with regard to smaller fires, and suggested that differences in the background characterisation of fire of $1 \mathrm{~K}$ can produce fire attribution errors of a factor of 100 or more. Issues also occur with deriving the background temperature from adjacent pixels. In some cases, as highlighted by (Giglio and Schroeder, 2014), estimation of the background temperature from adjacent pixels has produced a background temperature higher than the brightness temperature of the detected fire, leaving the fire's characteristics unable to be derived. These issues highlight the necessity of generating an accurate value for the background temperature. 
A method to circumvent this issue with regard to fire attribution is to derive the background temperature of the target pixel using a temporal based method to model a diurnal temperature cycle (DTC). This method uses a-priori knowledge of a pixel's DTC over a time period to project a background temperature of a target pixel if no affecting phenomenon was present. With the addition of geostationary sensors in recent years, the ability to utilise algorithms with a temporal component for land surface temperature (LST) determination has improved. Modelling of the DTC for LST from geostationary images has been undertaken using absolute descriptive models (Göttsche and Olesen, 2001, Jiang et al., 2006), Reproducing Kernel Hilbert Space models (van den Bergh et al., 2006, Udahemuka and Bergh, 2008), Fourier analysis (van de Kerchove et al., 2013), Artificial Neural Networks (Voyant et al., 2014) and Kalman filtering (Masiello et al., 2013, van den Bergh et al., 2009). Utilisation of a single value decomposition (SVD) method, such as that proposed in (Black and Jepson, 1998), has been applied by (Udahemuka and Bergh, 2008) and extended by (Roberts and Wooster, 2014) as part of a robust matching algorithm with significant improvements in the ability to handle temperature anomalies in the observation data.

\subsection{Robust Matching Algorithm}

This study make use of the robust matching algorithm described in (Roberts and Wooster, 2014), which adapted a form of single value decomposition described in (Black and Jepson, 1998) inclusive of a method to reduce the influence of outliers on the derived DTC. This method isolates 10 contamination-free DTCs in a 30-day period for use in a SVD, which, depending on the location, prevailing weather conditions and persistent thermal anomalies, may not always be achievable for every pixel in a dataset. (Roberts and Wooster, 2014) utilised a database of contaminationfree DTCs to populate the training dataset of a pixel which suffered from a deficit of its own DTCs for the SVD process. Depending on the number of supplemental DTCs used, coupled with the potential variation of the supplemental pixel's nominal DTC from the target pixel, using these supplemental DTCs to fill out an SVD could introduce error into the principal components of the DTC defined by the SVD, with subsequent errors in the fitting process.

\subsection{Wide Area DTC Derivation}

A brightness temperature return in the medium wave infra-red consists of two main components - reflection of solar radiation from the earth's surface, and emission of blackbody radiation from the surface due to temperature. The skin surface temperature of the earth is influenced by a number of factors - rain lowers surface temperatures very effectively, convective cooling and heating due to air masses can influence surface temperatures, and percentage land cover can affect the magnitude of temperature change - but the most notable influence is heating by solar radiation.

For an area of given latitude, the influence of solar radiation in both reflection from the surface and the heating of the surface should be similar for a time period given that these areas receive similar amounts of solar radiation (Bojanowski et al., 2014). The method outlined in this paper seeks to utilise this relationship to derive a wide area training DTC dataset for a band of latitude, which can then be applied to raw temperature data from pixels within this band of latitude to produce a fit for the DTC on a given day.

\section{METHOD}

\subsection{Derivation of Training Data}

To provide an estimate of a DTC for a specific day we will look to gather information about the pixel's ideal DTC behaviour in the previous seven days. This method will focus primarily on Band 7 brightness temperatures produced from AHI, as positive anomalies in this band are generally indicative of fire. The brightness temperature values for a pixel are extracted from the day's images, and these brightness temperatures are standardised for the day $($ mean $=0$, standard deviation $=1)$. The pixel's latitude and longitude are used to provide both the latitude of the estimated DTC band and the time offset from this series that needs to be applied to align local solar noon with the estimated solar noon, as in Eq. 1:

$$
t_{s}=U T C_{\text {image }}+\text { longitude } \times 240(\text { secs })
$$

The dataset this method has been applied to consists of the continent of Australia. The width of the landmass varies up to a maximum of $39^{\circ}$ of longitude, approximately corresponding to two hours and twenty minutes of elapsed time. For this study, a cell size of $0.25^{\circ}$ has been chosen, meaning that each cell roughly corresponds to one minute of solar time difference from an adjacent cell on the same latitude. If the maximum number of AHI images are available to be used in this process, around 13 estimates of the local temperature at solar time $t_{s}$ can be utilised in the broadest areas of the Australian continent.

For each image in the training data set, an ocean mask is applied to the original image, the brightness temperatures of pixels corresponding to each $0.25^{\circ}$ cell are grouped together, pixel values below $270 \mathrm{~K}$ are discarded to eliminate opaque clouds, and a median value is taken as an aggregation of the remaining non-cloud pixel values. The cell median temperatures are standardised in a similar way to the pixel for each day in the training data set. Each of the cells at each image is then assigned an apparent solar time, as in Eq. 1. With this time value assigned cells in the latitudinal set can be related to each other. At this stage all cells in the latitudinal band for all images in the set are merged together. As there are generally multiple returns at each time $t_{s}$ another median is taken to provide one value for each time in the series. A Butterworth low pass filter with a cut off frequency of six hours is then applied to the time series to eliminate standing variations in the training data.

\subsection{SVD of individual pixels}

In a similar fashion to the training data set, each pixel brightness temperature value in the DTC is assigned an apparent solar time given by Eq. 1. These times are rounded to the nearest minute to more easily correspond with the training dataset. The time offset between the first brightness temperature measurement of the pixel and the training data is calculated, and the values are extracted from the training dataset corresponding to the image times of the pixel dataset. This leaves the training dataset consisting of a number of daily vectors that correspond directly to the times that brightness temperatures were measured for the pixel's DTC.

A single value decomposition decomposes the training data matrix $A$ into a number of principal component vectors $U$ which describe the training data as a series of orthogonal vectors, along with the diagonal matrix $\Sigma$ which contains sorted decreasing eigenvalues for each component, and the matrix $V$ which contains coefficients for the reconstruction of the basis DTCs, as shown in 
Eq. 2:

$$
A=U \Sigma V^{T}
$$

For a vector of observations from a pixel $e$, an approximation of the DTC $e^{*}$ can be created from a reconstruction of the prinicpal components:

$$
e^{*}=\sum_{i=1}^{K} c_{i} U_{i t}
$$

where $K$ is the number of principle components used and $c_{i}$ is a series of scalar values derived by taking the inner product of the observations from the pixel and the principle components $\left(e^{T} U\right)$. $c_{i}$ essentially describes the contribution of each of the component vectors in $U$ to the fitted estimate of the DTC $e^{*}$.

At this point, to minimise the effects of outliers on the robust determination of the DTCs, a least squares minimisation of Eq. 3 occurs utilising a robust error norm, which identifies outliers that contribute excessively to error:

$$
\sigma(x, s)=\frac{x^{2}}{\sigma+x^{2}}
$$

$x$ is the value of $e-e^{*}$ at time $t$, and $\sigma$ is a scale factor which can be reduced iteratively to improve the solution given by the error minimisation. This can lead to the application of an outlier mask $m$, such that:

$$
m_{t}=\left\{\begin{array}{l}
1,\left|e_{t}-e_{t}^{*}\right| \geq \sigma / \sqrt{3} \\
0, \text { otherwise }
\end{array}\right.
$$

which leads to the minimisation of the error function $E(c)$ as shown below:

$$
E(c)=\sum_{t=1}^{N} m_{t} \rho\left(\left(e_{t}-\left(\sum_{i=1}^{K} c_{i} U_{i t}\right)\right), \sigma\right)
$$

This process refines the values of $c_{i}$ applied to the principal components $U_{i}$ in order to reconstruct an approximate value for $e_{t}$. At this point outliers from the ideal DTC can be identified and attributed according to their characterstics - whether they area positive or negative anomalies, and whether the change occurs suddenly or gradually over time. The idealised DTC curve can also be subsequently utilised as a state vector for a Kalman filter estimation of DTC for the pixel over subsequent days.

\section{CASE STUDY: ESPERANCE REGION FIRES}

On the 15 th November 2015, a fire caused by lightning strikes started in scrub forest near the locality of North Cascade in the Esperance region, around 700km ESE of Perth, Western Australia (location shown in Figure 1). Two days later on the 17th November, the fire broke containment lines during one of the worst days in recorded history for fire danger in Australia. The fire spread across low scrub forest and wheat fields driven by hot, strong north-westerly winds, stretching almost $90 \mathrm{~km}$ in length in a matter of hours, before a weak cool change from the south west slowed the fire's spread significantly. An unrelated fire was also burning to the immediate east of the town of Esperance in Merivale, but being constrained by the coast it did not exhibit the intensity or rapid growth of the North Cascade fire.

In figure 2, visual inspection of the medium wave infra red Band 7 image from AHI shows the extents and intensity of the later stages of the fire. The fire at this point covered around 1800ha, according to estimates of DFES, the state emergency services organisation. At several points during the fire's spread, the intensity

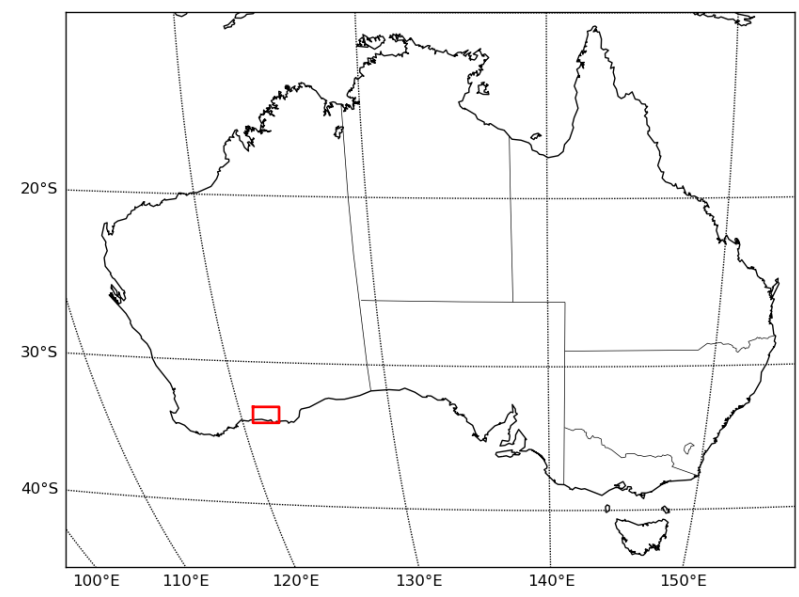

Figure 1: Location of case study area, Esperance region, Western Australia

and subpixel area of the fire was such that the Band 7 sensor was saturated, which considering the $400 \mathrm{~K}$ saturation temperature of the AHI sensor in this band demonstrates the coverage and intensity of the fire. This fire is significant not only for its intensity and spread rate, but also for the strong smoke output driven by the fire, which caused occlusion of land temperatures ahead of the North Cascade fire and of the fire output of the Merivale fire, which was partially downwind of the main complex. This smoke plume is made evident by the corresponding Band 13 thermal image, with the smoke plume measuring up to $30 \mathrm{~K}$ lower than the surrounding surface returns.

Given the extreme weather conditions, the remoteness of the ignition point of the fire and the subsequent inability to monitor the fire closely from the surface, the AHI images provide regular updates of the fire spread throughout the day. Secondary metrics such as rate of spread and fire radiative output are easily derived once an ignition temperature threshold is defined.

To investigate the robust fitting technique, we applied it to a number of pixels both within the fire region and surrounding it for the day prior to the fire and the day of the fire. In figure 3 , the robust fitting algorithm reflects the observations well for the anomaly free day on the 16th November, but shows distinct issues with fitting the DTC on the 17th. The normal background on this day suffers from significant occlusion from around midday local solar time due to smoke passing overhead, depressing brightness temperatures by up to $10 \mathrm{~K}$ below the estimated temperature. This sustained negative anomaly flips around 0815 UTC when the area catches alight, and sustained positive brightness temperature anomalies of up to $12 \mathrm{~K}$ persist until fire activity subsides with the full onset of a wind change around 1100 UTC. The robust fitting algorithm in this case has overfitted to the diminished smoke affected temperature values, underestimating the surface temperature by up to $10 \mathrm{~K}$. The dashed line here shows the first estimate of $c_{i}$ using only the primary component of the SVD, which shows inflation from the high temperatures detected during the fire, but nevertheless follows the understood relationship of the DTC shape. Of significance here also to the fitting of the DTC is the change in temperature response to sunlight on the morning after the fire. The accelerated rate of temperature increase is due to the decreased albedo of the surface after the fire has passed, and this relationship can be discerned from the brightness temperature response in the thermal band.

At this point we can create a crude map of fire spread for the day using a basic threshold algorithm. A conservative figure for 

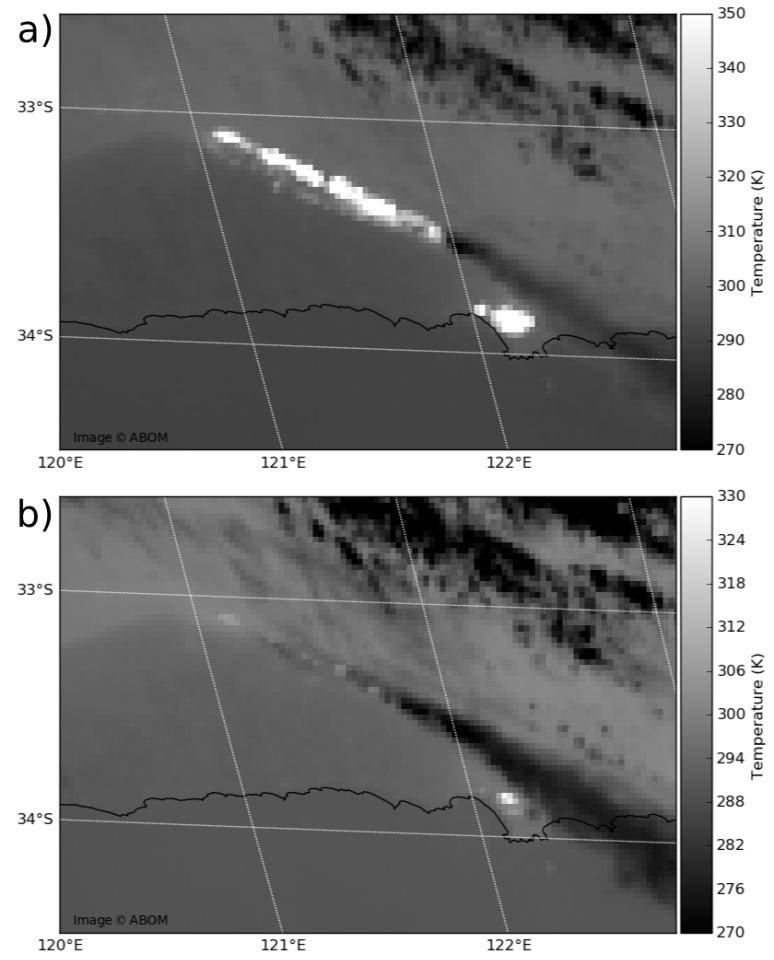

Figure 2: AHI- 8 brightness temperature image of the North Cascade and Merivale fires, Esperance region of Western Australia, 2015-11-17 1000 UTC (1800 AWST). a) Band 7 - 3.9 $\mu \mathrm{m}$ b) Band $13-10.8 \mu \mathrm{m} \odot \mathrm{ABOM} 2015$

an ignition threshold could be considered as a brightness temperature of an observation which is $5 \mathrm{~K}$ above the fitted DTC. Figure 4 shows the time where Band 7 brightness temperatures first reach this mark in pixels that recorded at least one brightness temperature greater than $335 \mathrm{~K}$ on the 17 th November. The North Cascade fire shows the expected behaviour early in the day, with flaming pixels at the source detected early, and the initial spread mapped well. Issues with the fitting of smoke to the modelled DTC become apparent here with the false early returns south east of the fire - these are most likely due to gaps in the smoke showing the true background temperature at these times and causing large positive anomalies. The figure also demonstrates the wind change later in the day, with the fire growing in a north easterly direction later in the day. The Merivale fire is also shown here, and due to the local geography there is little deviation to the fire extent with the wind from the north west. This fire finally sees some growth late in the day with the south easterly change in wind. Note that fire temperatures above $335 \mathrm{~K}$ are being seen in pixels where the majority of the pixel is water - this suggests that the sensor may have some contamination of adjacent pixels from a point spread effect. More analysis will be required to determine the magnitude of this effect especially in coastal areas.

\section{DISCUSSION}

Overall, the sensor bands covered in this investigation look to adequately characterise fire in its early stages. Given that this case is a rather extreme example, with weather conditions increasing background temperatures well beyond their usual values for this latitude, coupled with low humidity and dry fuel providing ideal conditions for fire spread, the extent and intensity of this event are easy to detect and monitor. This fire also look place in a largely

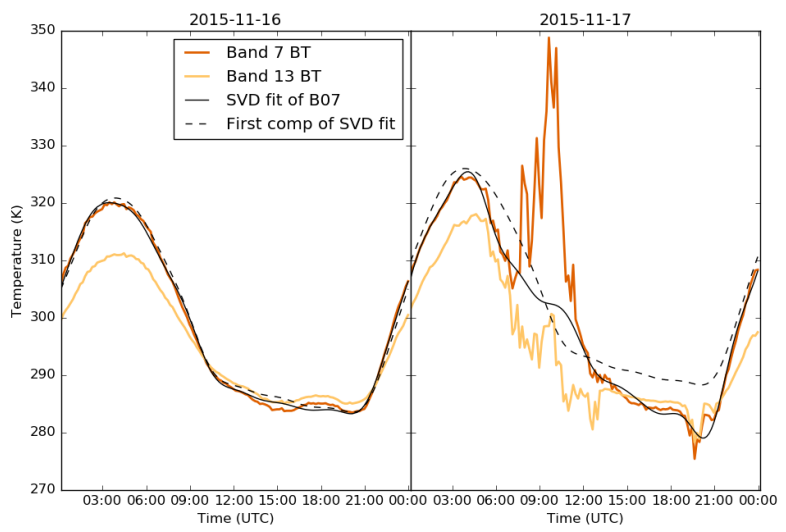

Figure 3: Robust matching algorithm results for a typical pixel (-35.2583S, 121.4374E) in the North Cascade complex fire 201511-16 and 17.

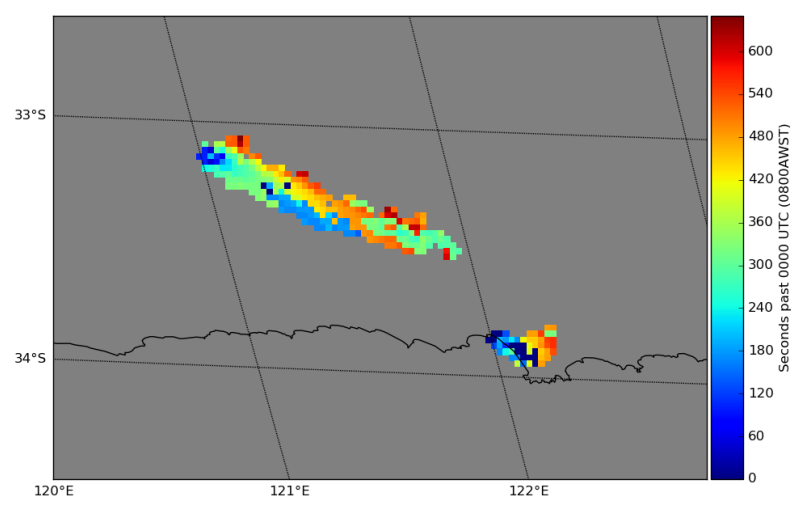

Figure 4: Map of first positive anomaly return for calendar day 2015-11-17 for pixels which recorded a brightness temperature greater than $335 \mathrm{~K}$ at any time.

open landscape - the area generally consists of wheatfields and grassland, interspersed with low woody scrubland which is of limited height. Investigations should be made into the initial detection of fires which there may be significant in-situ obstacles to imaging, such as vegetative canopy cover.

Another element of interest highlighted by this investigation is the effect of smoke on brightness temperatures in the areas adjacent to fire. Particulate matter in the smoke tends to reduce the amount of solar radiation reaching the surface(Tarasova et al., 1999), along with scattering the medium wave infrared reflecting from the surface. Fire activity also increases the turbidity of the atmosphere (Kumar et al., 2010), increasing the opacity of the atmosphere resulting in reduced brightness temperatures on the leeward side of fire events. The presence of smoke particulate in the atmosphere also tends to attract moisture to condensate and form clouds, exacerbating scattering further. The reduction of solar radiation reaching the surface can also contribute to a drop in surface temperature and subsequent blackbody radiance of the surface.

The propensity of smoke to lower brightness temperatures in the area immediately downwind of a fire leads to the misattribution of fire background temperatures when a contextual algorithm is used for fire detection. The exact influence of smoke on medium wave brightness temperatures is poorly known - studies on smoke oc- 
clusion have tended to focus on visible and near infra-red wavelengths. The ability of AHI-8 to provide rapid updates of brightness temperature changes downwind of a fire, coupled with insitu sensors of downwelling and upwelling radiance, could provide greater understanding of the effects of smoke on longer wavelength radiation, and subsequently improve the ability to detect fires where aerosol characteristics are known.

The sensor shows great promise with regard to mapping the evolving nature of a fire event. The target fire is quite intense in nature, and coupled with the open landscape, the initial ignition of fires is easy to discern from initial investigations of imagery. The methods used in this paper to map the extents of the fire's change over time are very crude, and associated issues with smoke and rapid temperature change have manifested in the results derived. Application of method such as that demonstrated in (Diagne et al., 2010), where differencing of the medium wave and thermal infrared temperatures is used in conjunction with basic thresholds, should reduce the commission errors in the fitted model caused by spikes such as smoke boundaries.

The use of this adaption of the robust fitting method presented in this study for fire background determination has some advantages over previous implementations. Using training DTCs from a thirty day period increases errors in defining the shape of the modelled DTC due to factors such as the solar geometry changes over the time period, changing both the length of day and the total solar radiation input into the system. The precession of the earth's orbit can also lead to changes in the timing of peak solar radiation for the day. The error budget created by these factors can be minimised by reducing the number of training DTCs used to calculate the modelled DTC, at the risk of introducing errors through a lack of non-cloud influenced training data. In some places it may be impossible to obtain training DTC profiles, such as where there is prevailing cloud cover for significant portions of the training data window, or where there are periodic daily cycles of cloud formation which cause attribution issues during that window. By choosing an aggregation method to create the DTC model, the amount of days required to be sampled can be decreased to get a finer resolution for the precise modelled DTC with less noise than the individual pixel method.

Using the method presented in this paper provides an anomaly free quantification of the typical response curve of a pixel contained within a small area of the earth's surface. This is of course not without its shortcomings. The method does not take into account variations in surface emissivity from pixel to pixel. The land surface emissivity can have a significant effect on the radiance emitted from an area given a known temperature. Correction for this could occur using a current model for land surface emissivity from another satellite sensor, or work could be completed to adapt a land surface emissivity model for the AHI sensor, at which point a pixel by pixel correction to the modelled DTC could be applied. This correction would have to be generated on a regular basis to account for land use and seasonal change in the environment.

Using this more aggregated model for DTC also eliminates the ability of the robust fitting algorithm to adjust to standing variations in a pixel from an ideal candidate. These could include such things as periodic cloud formation due to weather and topography, and shadowing of the pixel from solar radiation due to topography and land cover. Further investigation is required to identify areas that may be affected by such standing anomalies and steps than can be taken to mitigate their effects on overall fire detection accuracy.

Formation of the model is also dependent on the landforms present in the latitudinal band under consideration. The minimum width of land required to obtain a solution across a full disk image is $2.5^{\circ}$ of longitude, and widths of less than $5^{\circ}$ of longitude reduce redundancy of measurements and can lead to standing errors in the resultant modelled DTC. Creating cells further away from the satellite nadir can overtly influence the modelled result as well the limited number of pixel returns from the cell at the edge of a full disk can introduce biases especially at times where sun glint may elevate resultant brightness temperatures.

No correction has been applied for sun glint to this model in this study, which may cause heightened brightness temperature returns in the medium wave infra red at times where the sensor viewing angle coincides with the specular reflectance of the sun from a horizontal surface, and a correction such as that proposed by (Zhukov et al., 2006) could be applied to each pixel identified as a potential target.

There is significant scope for the expansion of methods used to quantify the land surface temperature included in this paper. The use of a single value decomposition method for the robust fitting method tends to amplify the effect of the secondary eigenvectors in determining the DTC for a day. The single value decomposition process identifies the general shape of the DTC very well within the first eignevector, with subsequent orthogonal vectors having eigenvalues generally twenty times lower. Using the weighted norm to reconstruct the DTC tends to treat the influence of the eigenvectors as uniform, and as such tends to provide overfitted solutions for DTCs on days with large number of cloud and smoke affected pixels. Subsequent work should investigate the use of a technique such as parallel factor analysis such at that discussed in (Abdi et al., 2013), which takes into account the strength of each fitted component in a factored solution. Notwithstanding this, the likelihood of this method providing improved results for the generation of DTCs over a more dynamic method such as Kalman filtering is low, and whilst refinements can be made, this method for determining thermal anomalies works best as a starting point for a filtering based solution.

\section{ACKNOWLEDGEMENTS}

The support of the Commonwealth of Australia through the Cooperative Research Centre program is acknowledged. The authors would also like to acknowledge the support of the Australian Bureau of Meteorology, Geoscience Australia, and the Japanese Meteorological Agency for use of AHI imagery associated with this research, along with the Australian National Computational Infrastructure for their support with data access and services.

\section{REFERENCES}

Abdi, H., Williams, L. J. and Valentin, D., 2013. Multiple factor analysis: principal component analysis for multitable and multiblock data sets. Wiley Interdisciplinary Reviews: Computational Statistics 5(2), pp. 149-179.

Black, M. and Jepson, A., 1998. Eigentracking: Robust matching and tracking of articulated objects using a view-based representation. International Journal of Computer Vision 26(1), pp. 63-84.

Bojanowski, J. S., Vrieling, A. and Skidmore, A. K., 2014. A comparison of data sources for creating a long-term time series of daily gridded solar radiation for Europe. Solar Energy 99, pp. 152-171.

Diagne, M., Drame, M., Ferrão, C., Marchetti, P. G., Pinto, S. and Rivolta, G., 2010. Multisource data integration for fire risk management: The local test of a global approach. IEEE Geoscience and Remote Sensing Letters 7(1), pp. 93-97. 
Dozier, J., 1981. A method for satellite identification of surface temperature fields of subpixel resolution. Remote Sensing of Environment 11(C), pp. 221-229.

Giglio, L. and Kendall, J. D., 2001. Application of the Dozier retrieval to wildfire characterization a sensitivity analysis. Remote Sensing of Environment 77(1), pp. 34-49.

Giglio, L. and Schroeder, W., 2014. A global feasibility assessment of the bi-spectral fire temperature and area retrieval using MODIS data. Remote Sensing of Environment 152, pp. 166-173.

Göttsche, F.-M. and Olesen, F. S., 2001. Modelling of diurnal cycles of brightness temperature extracted from METEOSAT data. Remote Sensing of Environment 76(3), pp. 337-348.

Jiang, G. M., Li, Z. L. and Nerry, F., 2006. Land surface emissivity retrieval from combined mid-infrared and thermal infrared data of MSG-SEVIRI. Remote Sensing of Environment 105(4), pp. 326-340.

Kaufman, Y. J., Justice, C. O., Flynn, L. P., Kendall, J. D., Prins, E. M., Giglio, L., Ward, D. E., Menzel, W. P. and Setzer, A. W., 1998. Potential global fire monitoring from EOS-MODIS. Journal of Geophysical Research-Atmospheres 103(D24), pp. 3221532238.

Kumar, K. R., Narasimhulu, K., Balakrishnaiah, G., Reddy, B. S. K., Gopal, K. R., Reddy, R., Satheesh, S., Moorthy, K. K. and Babu, S. S., 2010. A study on the variations of optical and physical properties of aerosols over a tropical semi-arid station during grassland fire. Atmospheric Research 95(1), pp. 77-87.

Li, Z.-L., Tang, B.-H., Wu, H., Ren, H., Yan, G., Wan, Z., Trigo, I. F. and Sobrino, J. a., 2013. Satellite-derived land surface temperature: Current status and perspectives. Remote Sensing of Environment 131, pp. 14-37.

Masiello, G., Serio, C., De Feis, I., Amoroso, M., Venafra, S., Trigo, I. F. and Watts, P., 2013. Kalman filter physical retrieval of surface emissivity and temperature from geostationary infrared radiances. Atmospheric Measurement Techniques 6(12), pp. 3613-3634.

Roberts, G. and Wooster, M. J., 2014. Development of a multitemporal Kalman filter approach to geostationary active fire detection \& fire radiative power (FRP) estimation. Remote Sensing of Environment 152, pp. 392-412.

Roy, D. P., Boschetti, L. and Smith, A. M., 2013. Satellite Remote Sensing of Fires. In: Fire Phenomena and the Earth System: An Interdisciplinary Guide to Fire Science, pp. 77-93.

Tarasova, T. A., Nobre, C. A., Holben, B. N., Eck, T. F. and Setzer, A., 1999. Assessment of smoke aerosol impact on surface solar irradiance measured in the Rondônia region of Brazil during Smoke, Clouds, and Radiation - Brazil. Journal of Geophysical Research: Atmospheres 104(D16), pp. 19161-19170.

Udahemuka, G. and Bergh, F. V. D., 2008. Robust fitting of diurnal brightness temperature cycle. South African Computer Journal 40, pp. 1-6.

van de Kerchove, R., Lhermitte, S., Veraverbeke, S. and Goossens, R., 2013. Spatio-temporal variability in remotely sensed land surface temperature, and its relationship with physiographic variables in the Russian Altay Mountains. International Journal of Applied Earth Observation and Geoinformation 20(1), pp. 4-19.

van den Bergh, F., Udahemuka, G. and van Wyk, B. J., 2009. Potential fire detection based on Kalman-driven change detection. In: International Geoscience and Remote Sensing Symposium (IGARSS), Vol. 4, pp. 77-80. van den Bergh, F., van Wyk, M. A. and van Wyk, B. J., 2006. Comparison of data-driven and model-driven approaches to brightness temperature diurnal cycle interpolation. 17th Annual Symposium of the Pattern Recognition Association of South Africa.

Voyant, C., Haurant, P., Muselli, M., Paoli, C. and Nivet, M. L., 2014. Time series modeling and large scale global solar radiation forecasting from geostationary satellites data. Solar Energy 102, pp. 131-142.

Wooster, M. J., Zhukov, B. and Oertel, D., 2003. Fire radiative energy for quantitative study of biomass burning: Derivation from the BIRD experimental satellite and comparison to MODIS fire products. Remote Sensing of Environment 86(1), pp. 83-107.

Zhukov, B., Lorenz, E., Oertel, D., Wooster, M. and Roberts, G., 2006. Spaceborne detection and characterization of fires during the bi-spectral infrared detection (BIRD) experimental small satellite mission (2001-2004). Remote Sensing of Environment 100(1), pp. 29-51. 